\begin{tabular}{|c|c|}
\hline Title & $\begin{array}{l}\text { Neutron spectrum change with thermal moderator temperature in a compact electron accelerator-driven neutron source } \\
\text { and its effects on spectroscopic neutron transmission imaging }\end{array}$ \\
\hline Author(s) & Ishikawa, Hirotaku; Kai, Tetsuya; Sato, Hirotaka; Kamiyama, Takashi \\
\hline Citation & $\begin{array}{l}\text { Journal of nuclear science and technology, 56(2), 221-227 } \\
\text { https://doi.org/10.1080/00223131.2018.1561339 }\end{array}$ \\
\hline Issue Date & 2019-02-01 \\
\hline Doc URL & http:/hdl.handle.net/2115/76641 \\
\hline Rights & $\begin{array}{l}\text { This is an A ccepted Manuscript of an article published by Taylor \& Francis in Journal of nuclear science and } \\
\text { technology on 01 Feb 2019, available online: http://www.tandfonline.com/10.1080/00223131.2018.1561339. }\end{array}$ \\
\hline Type & article (author version) \\
\hline File Information & 87569(Journal_of_nuclear_science_and_technology_56_221-227).pdf \\
\hline
\end{tabular}

Instructions for use 


\title{
Neutron spectrum change with thermal moderator temperature in a compact electron accelerator-driven neutron source and its effects on spectroscopic neutron transmission imaging
}

\author{
Hirotaku Ishikawa $^{1 *}$, Tetsuya Kai ${ }^{2}$, Hirotaka Sato ${ }^{1}$ and Takashi Kamiyama ${ }^{1}$ \\ ${ }^{1}$ Graduate School of Engineering, Hokkaido University, Kita 13, Nishi 8, Kita-Ku, Sapporo, Hokkaido \\ 060-0828, Japan; ${ }^{2}$ Materials and Life Science Division, J-PARC Center, Japan Atomic Energy Agency, \\ 2-4 Shirakata, Tokai-mura, Naka-gun, Ibaraki 319-1195, Japan
}

Recently, Compact Accelerator-driven Neutron Sources (CANSs) are attracting attention. In CANSs, a simple thermal neutron moderator such as polyethylene is often used from the viewpoints of cost, simplicity and maintainability. In most cases, the temperature of such a moderator has not been controlled although it is natural that the moderator temperature and the neutron spectrum will change with accelerator-operation. Thus, we simultaneously measured neutron spectra and the temperature of a polyethylene moderator at the Hokkaido University Neutron Source (HUNS) driven by a compact electron accelerator to observe the effect of any temperature change on the reliability of spectroscopic transmission measurement. The ratio of the neutron effective temperature and the moderator temperature was constant in HUNS case, although both increased by 4-5 K within one hour after the start of accelerator-operation. This indicated that the neutron effective temperature was well estimated by the moderator temperature. The effect of the temperature change can be easily avoided by excluding data collection before the moderator warms up. These results suggested that the monitoring of moderator temperature is recommended in compact electron accelerator-driven neutron sources with a thermal 
neutron moderator to guarantee reliability of spectroscopic transmission measurement without sacrifices of cost, simplicity and maintainability.

Keywords: compact electron accelerator-driven neutron source; thermal neutron moderator; moderator temperature; neutron spectrum; polyethylene moderator; neutron transmission spectroscopy; effective neutron temperature; Maxwell-Boltzmann distribution 


\section{Introduction}

Low-energy neutrons have been used for material science and non-destructive inspection of light-elements in a bulk object by taking advantage of their high-penetration power in most of materials and their high sensitivity to light elements. Neutron sources are mainly driven by an accelerator or a research reactor. Accelerator-driven neutron sources have attracted attention over the last several decades. In particular, high-intensity neutron sources such as SNS[1], J-PARC[2] and ESS[3] have started operation or are being constructed. These are expected to be world-leading facilities for neutron applications. Additionally, interest is not only in such big facilities, but also in the development of Compact Accelerator-based Neutron Sources (CANSs) for use in small installations in universities and industrial locations, due to the shutting down of many of small research reactors in recent years[4]. In Japan, many CANSs have been developed[5]: Hokkaido University Neutron Source (HUNS)[6], Aomori Prefecture Quantum Science Center[7], Riken Accelerator-driven Neutron Source (RANS)[8], Kyoto University Accelerator-driven Neutron Source (KUANS)[9], Kyoto University Research Reactor Institute LINAC (KURRILINAC)[10] and Sumitomo Heavy Industry ATEX (SHI-ATEX)[11].

One of the simple application of CANSs is neutron radiography which visualizes the inside of an object. The radiography technique can be extended to visualize a distribution of crystalline or elemental information by analyzing the transmission spectra at each pixel of a two-dimensional detector coupled with the time-of-flight (TOF) technique and the pulse mode operation of an accelerator[12]. Only a time-analyzing neutron-imaging detector (for example neutron-GEM[13] and $\mu \mathrm{NID}[14]$ ) is required for such spectroscopic imaging technique. Such a simple experimental configuration is suitable in CANSs.

For cost, simplicity and maintainability, a simple thermal moderator made from roomtemperature polyethylene is often used in $\operatorname{CANSs}[6,8-11]$. In most cases, the temperature of the moderator has not been controlled for keeping simplicity and maintainability. 
Although it is natural that the moderator temperature increases or decreases with the accelerator operation, which causes shifts of a neutron spectrum, small attention has been paid to the effect. This was because high-precision measurements have not been in much demand so far. However, as the spectroscopic imaging technique develops, more precise measurements are needed. In particular, the effect of a spectral shift is a concern for spectroscopic transmission measurements since an energy spectrum from a sample needs to be divided by the spectrum without the sample, assuming the energy spectra are stable during the measurement. Thus, in this study, we measured the temperature of the room-temperature polyethylene moderator by a thermocouple in the Hokkaido University Neutron Source (HUNS) driven by compact electron accelerator, and simultaneously measured neutron spectra as a function of the elapsed time after the start of accelerator operation. An electron-accelerator-type CANS is the most suitable to estimate the effects of moderator temperature change since the heat deposition in a moderator in electronaccelerator-type CANS is higher than that of other types, e.g., the proton type. Based on the measured neutron energy spectra, the effects of temperature changes on neutron transmission-spectroscopy measurement and its analysis are discussed. The results allowed us to make guidelines for the design, operation and measurement in CANSs with simple room-temperature moderators, especially in electron-accelerator-type CANS.

\section{Experimental setup and data analysis method}

\subsection{Experimental setup}

The measurement of neutron spectra together with the temperature of a polyethylene moderator was carried out in the compact accelerator-driven thermal neutron source at Hokkaido University coupled with an electron linear accelerator. Figure 1 shows the experimental setup.

[Figure 1 about here.] 
There are two rooms: a target room for the neutron production and an experimental room for neutron measurements, separated by a $3.5 \mathrm{~m}$ thick concrete wall. In the target room, a target-moderator-reflector assembly is composed of a lead target (80 mm cube), a polyethylene coupled moderator (H $\left.120 \times \mathrm{W} 120 \times \mathrm{L} 50 \mathrm{~mm}^{3}\right)$ and a graphite reflector (0.5 $\mathrm{m}$ cube). The densities of the lead, the polyethylene and the graphite are 11.3, 0.94 and $1.8 \mathrm{~g} / \mathrm{cm}^{3}$, respectively. Horizontal beam paths of electrons and neutrons are provided in the assembly with a $100 \mathrm{~mm}$ square cross-section. The polyethylene coupled moderator is located at the same height as the target, and the neutron path is perpendicular to that of the electron beam. The moderator is composed of 4 polyethylene pieces that are H $60 \times$ W $120 \times$ L $25 \mathrm{~mm}^{3}$ in size. High energy neutrons are generated by the $(\gamma, \mathrm{n})$ reaction initiated by bremsstrahlung caused by electron beam injection to the lead target. The generated neutrons are moderated in the polyethylene moderator, and then thermal neutrons are delivered to the experimental room along the neutron beam path. For the present measurements, the accelerator was operated at $50 \mathrm{~Hz}$, and provided a $34 \mathrm{MeV}$ electron beam of $3 \mu \mathrm{s}$ in pulse width and $36 \mu \mathrm{A}$ in average current. In the experimental room, a neutron detector was set at $6.67 \mathrm{~m}$ from the moderator surface. The GEM (Gas Electron Multiplier) neutron imaging detector[13], in which enriched boron-10 (purity greater than 99\%) was utilized as a neutron converter, was used with an $\mathrm{Ar}-\mathrm{CO}_{2}(\mathrm{Ar}$ : $\left.\mathrm{CO}_{2}=70: 30\right)$ working gas. The applied voltage to the detector was $-3040 \mathrm{~V}$. The efficiency of the GEM detector was assumed to be proportional to the cross-section of the ${ }^{10} \mathrm{~B}(\mathrm{n}, \alpha)$ reaction.

In this experiment, the temperature of the polyethylene moderator was measured with a chromel-alumel (type-K) thermocouple. The thermocouple was attached on one of the four polyethylene pieces consisting the moderator by using a polyimide tape. The thermocouple was placed at $5 \mathrm{~mm}$ below the center of the moderator assembly. The temperature was recorded by a data logger (GRAPHTEC midi LOGGER GL240) at a 1- 
second intervals. Ice water in the Dewar flask was used to guarantee the zero degree Celsius reading of the thermocouple. Neutron spectra for open beam were measured by the GEM detector with the event recording mode, simultaneously. The temperature and neutron spectra were measured for 6 hours with the accelerator operating. After the measurement, the recorded temperature and the measured neutron spectra were averaged in 5 minutes interval before the data analysis. Additionally, the neutron transmission spectrum of an $\alpha$-iron plate was also measured for the calibration of the neutron flight-path length. The density of the $\alpha$-iron plate is $7.84 \mathrm{~g} / \mathrm{cm}^{3}$, and the size of that is $\mathrm{H} 200 \mathrm{x}$ W $100 \times \mathrm{L} 10$ $\mathrm{mm}^{3}$.

\subsection{Data analysis}

To evaluate the shift of the measured neutron spectra, effective neutron temperatures were derived by fitting the Maxwell-Boltzmann distribution, $f_{\mathrm{eq}}(E)$, as expressed in Equation (1), to measured neutron spectra integrated in 5 minutes interval in the thermal equilibrium region with a least-squares method.

$$
f_{\text {eq }}(E)=N \times 4 \pi v^{2}\left(\frac{\mathrm{m}}{2 \pi \mathrm{k} T}\right)^{3 / 2} \exp \left(-\frac{\mathrm{m} v^{2}}{2 \mathrm{k} T}\right)=N \times \frac{4 E}{\mathrm{k} T} \sqrt{\frac{\mathrm{m}}{2 \pi \mathrm{k} T}} \exp \left(-\frac{E}{\mathrm{k} T}\right) .
$$

Here $E$ is the neutron energy, $N$ the total number of neutrons, $T$ the effective neutron temperature, $\mathrm{m}$ the mass of a neutron, $\mathrm{k}$ the Boltzmann constant, and $v$ the neutron velocity. In the energy range above the thermal equilibrium region, the neutron spectra were fitted by $f_{1 / E}(E)$ described in Equation (2).

$$
f_{1 / E}(E)=\frac{1}{1+\exp (a / \sqrt{E}-b)} \times \frac{c}{E}\left(\frac{E}{E_{0}}\right)^{\alpha},
$$

where $a, b, c$ and $\alpha$ show fitting parameters, and $E_{0}$ is $1 \mathrm{eV}$. This equation is the product of the connecting function (the former part) and the $1 / E$ function (the latter part)[15]. Before the fitting analysis, we converted a neutron TOF spectrum, which was binned in a TOF width of $20 \mu \mathrm{s}$, to a neutron energy spectrum. Equation (3) shows the calibration 
equation converting a TOF to a neutron energy.

$$
E=\left(\frac{72.3 \times L}{t-t_{0}}\right)^{2}
$$

where $L$ is the neutron flight-path length in meters, $t$ the TOF in micro-seconds, $t_{0}$ the delay time in micro-seconds, and the units of $E$ is electron-volt. The values of $L$ and $t_{0}$ were determined from wavelength of the Bragg-edges in the neutron transmission spectrum of the $\alpha$-iron plate using the Bragg-edge analysis code, RITS[16-19]. In the analysis, we used the Bragg-edges in the range from 0.18 to $0.4 \mathrm{~nm}$, namely, from 0.005 to $0.02 \mathrm{eV}$. From the result of the analysis, $L$ and $t_{0}$ were deduced as $6.67 \mathrm{~m}$ and $78.1 \mu \mathrm{s}$, respectively.

Figure 2 shows an example of the fitting analysis to obtain an effective neutron temperature.

[Figure 2 about here.]

In the upper graph of Fig. 2, a measured neutron energy spectrum is shown by dots with error bars, and a result of fitting is shown by a line. The difference of the measured and fitting result is shown in the lower. The statistical errors of the each dot were smaller than $1 \%$ above the energy range of $0.01 \mathrm{eV}$, and the systematic error which was mainly caused by background noise, e.g., environmental neutron and electric noise of the detector, was smaller than $1 \%$ in the energy range of $0.01-10 \mathrm{eV}$. The systematic error was estimated from the ratio of neutron counts in the measurement with and without a neutron stopper in front of the detector. The neutron stopper was the mixture block of boric acid and resin material. The sum of equations (1) and (2) was used as the fitting function, and the fitting range was $0.01-10 \mathrm{eV}$. The number of the fitting parameters was $6: N, T, a, b$, $c$, and $\alpha$. Before the fitting analysis, initial values of the 6 parameters were determined in advance by pre-fitting analysis. Firstly, initial values of $N$ and $T$ were determined by fitting the equation (1) to the measured neutron energy spectrum in the energy range between $0.01-0.07 \mathrm{eV}$. Secondly, initial values of $c$ and $\alpha$ were determined by fitting the $1 / E$ function in the equation (2) to the measured spectrum in the energy range between 
$0.5-10 \mathrm{eV}$. Finally, initial values of $a$ and $b$ were determined by the fitting the sum of the equations (1) and (2) to the measured spectrum in the energy range between $0.01-10 \mathrm{eV}$, with the condition that the obtained initial values of $N, T, c$ and $\alpha$ were assigned and fixed. After determination of the initial values of all parameters, the fitting analysis was performed by fitting the sum of the equations (1) and (2) to the measured spectrum in the energy range between $0.01-10 \mathrm{eV}$, with the condition that the obtained initial values of all parameters were assigned and unfixed. From the results of the fitting analysis, the value of $T$ was obtained. In the upper graph of Fig. 2, the fitting curve did not match the measured spectrum below $0.01 \mathrm{eV}$. This is caused by that neutrons in the moderator leaked outside before reaching thermal equilibrium since the volume of the moderator is finite, and that more neutrons are absorbed by the moderator in the lower energy range since the absorption cross-section becomes larger in the lower energy range[20]. Note that the value of $T$ is generally higher than the temperature of the moderator due to the same reasons above.

In the lower graph of Fig. 2, there were some difference between the measured and fitting spectra above $0.01 \mathrm{eV}$. However, the value of $T$ was properly obtained, because the value of $T$ was approximately determined from the peak position of a measured spectrum, and because the fitting curve, namely, the Maxwell-Boltzmann distribution almost matched the measured one in the vicinity of the peak position $(0.02-0.04 \mathrm{eV})$.

\section{Results and discussion}

Figure 3 shows measured neutron energy spectra when the moderator temperature, $T_{\text {moderator }}$, was at two different temperatures.

[Figure 3 about here.]

The dashed line shows the spectrum which was measured during the first 5 minutes from the measurement start (the averaged $T_{\text {moderator was }} 298.2 \mathrm{~K}$ during that time), and 
the solid line shows the spectrum which was measured during the last 5 minutes (the averaged $T_{\text {moderator }}$ was $302.9 \mathrm{~K}$ ). The statistical errors of the two spectra were smaller than $1 \%$ above the energy range of $0.01 \mathrm{eV}$, and the systematic error mainly caused by the background noise was also smaller than $1 \%$ in the energy range of $0.01-10 \mathrm{eV}$. The two spectra in Fig. 3 look very similar. However, the actual shift of the spectrum with the change of $T_{\text {moderator }}$ was found by analyzing measured neutron spectra with the fitting analysis. Figure 4 shows the time evolution of the effective neutron temperatures, $T_{\text {neutron }}$, which represents the shift of the spectrum, and $T_{\text {moderator }}$ as a function of accelerator operation time. The ratio of the temperatures is also plotted.

[Figure 4 about here.]

$T_{\text {neutron }}$ was obtained by applying the fitting analysis to the neutron spectra which were measured every 5 minutes. The statistical and systematic errors of all measured spectra were smaller than $1 \%$ in the fitting energy range. The errors of $T_{\text {neutron }}$ in the fitting analysis were about $0.2 \mathrm{~K}$ in all plots. Although it was considered that temperature distribution existed in the inside of the moderator, we assumed homogeneous distribution in this analysis. $T_{\text {moderator }}$ was obtained by averaging thermocouple values every 5 minutes. The standard deviations of $T_{\text {moderator }}$ were below $0.4 \mathrm{~K}$ in the plots within an hour after the accelerator operation starts, and those after an hour were about $0.05 \mathrm{~K}$. The systematic error of the type-K thermocouple can be caused by calibration error, and the degree of the error in the type-K thermocouple was defined within $\pm 2.5 \mathrm{~K}$ at around the room-temperature in JIS (Japanese Industrial Standards) C 1602:2015[21]. $T_{\text {neutron was }}$ constantly larger than $T_{\text {moderator }}$ because the peak of the measured spectrum shifts to higher energy compared to that of the Maxwell-Boltzmann distribution at $T_{\text {moderator }}$, due to the leakage of the neutron from the moderator and neutron absorption by the moderator, as was mentioned in Sec. 2.2. In Fig. 4, $T_{\text {moderator }}$ increased by $4-5 \mathrm{~K}$ within an hour after the accelerator operation starts, and then became almost constant (only a $1 \mathrm{~K}$ 
further increase was apparent). $T_{\text {neutron }}$ also increased with the same trend as $T_{\text {moderator }}$, and the ratio of $T_{\text {neutron }}$ and $T_{\text {moderator }}$ was unchanged even though both of the temperatures increased. It indicated that the spectrum shifted with the change of $T_{\text {moderator }}$, and that the stability of $T_{\text {neutron }}$ was reflected by $T_{\text {moderator }}$. As to the cause of increasing $T_{\text {moderator }}, \gamma$-heating and the heat generated through the neutron-moderation process were considered.

It is likely that the shift of a neutron spectrum, represented as the change of $T_{\text {neutron }}$, could bring about undesirable effects in neutron applications, particularly in the case that requires precise measurements. To discuss this effect, we simulated neutron transmission spectrum incorporating the change of $T_{\text {moderator }}$, and compared it with the ideal, for example. In the transmission spectroscopy method, a neutron transmission spectrum of a sample, $\operatorname{Tr}(E)$, is calculated by equation (4),

$$
\operatorname{Tr}(E)=\frac{I(E)}{I_{0}(E)}
$$

where $I(E)$ is a measured neutron energy-dependent intensity through a sample, $I_{0}(E)$ is that without a sample. $I(E)$ is theoretically calculated by equation (5),

$$
I(E)=I_{0}(E) \times \exp (-N \sigma)
$$

where $N$ represents the areal atomic density of a sample and $\sigma$, the total cross-section of the sample. The neutron transmission spectroscopy method obtains information about a sample, e.g., the areal density and crystalline structure by analyzing $\operatorname{Tr}(E)$, assuming the neutron spectra are consistent, namely $T_{\text {moderator }}$ does not change, during the measurements. To simulate the $\operatorname{Tr}(E)$ assuming inconsistent neutron spectra, the measured neutron spectra at $T_{\text {moderator }}$ of $298.2 \mathrm{~K}$ and that of $302.9 \mathrm{~K}$, shown in Fig. 3, were used. We simulated the $\operatorname{Tr}(E)$ of an $\alpha$-iron of the thickness of $5 \mathrm{~mm}$. The cross-section of the iron sample was calculated by the RITS code. We obtained $I(E)$ of equation (4) by substituting $I_{0}(E)$ with a measured neutron spectrum at $T_{\text {moderator }}=302.9 \mathrm{~K}$ in equation 
(5), while $I_{0}(E)$ in equation (4) was measured one at $T_{\text {moderator }}=298.2 \mathrm{~K}$. Note that the neutron spectra were normalized by the integrated neutron counts above $1 \mathrm{eV}$, where the effect of the change of $T_{\text {moderator }}$ is negligible, to correct instabilities of detector gain, neutron intensity (caused by electron beam intensity and injection position on the target) etc. Figure 5 shows the simulated $\operatorname{Tr}(E)$, designated by dots and error bars, and the theoretical one designated by a solid line.

[Figure 5 about here.]

In the energy range from 0.05 to $0.3 \mathrm{eV}$, the simulated $\operatorname{Tr}(E)$ was greater than the theoretical one (by $\sim 1.5 \%$ at $0.12 \mathrm{eV}$ ). On the other hand, $\operatorname{Tr}(E)$ was smaller below 0.05 $\mathrm{eV}$ (by $\sim 3 \%$ at $3 \mathrm{meV}$ ).

The $4.7 \mathrm{~K}$ change of $T_{\text {moderator }}$ caused the shape-change of $\sim 3 \%$ at maximum in the neutron transmission spectra. For discussions about the shape-change effect on spectroscopic transmission analysis, we performed the Bragg-edge analysis for the simulated $\operatorname{Tr}(E)$ as an example. In the Bragg-edge analysis with the RITS code, crystalline structure information, e.g., lattice spacing, crystallite size and texture, was mainly obtained[16-18]. The analyzed values of the three parameters from the simulated $\operatorname{Tr}(E)$ were compared to those from the theoretical $\operatorname{Tr}(E)$. From the result of the Bragg-edge analysis, it was found that lattice spacing was not affected because the appearance energies of Bragg-edges were not affected by the $4.7 \mathrm{~K}$ change of $T_{\text {moderator }}$. On the other hand, it was found that other two parameters were affected by the $4.7 \mathrm{~K}$ change of $T_{\text {moderator }}$. In particular, the crystallite size was strongly affected, and the difference of the analyzed values reached to about $20 \%$. In an example of the crystallite size analysis[22], the crystallites of welded and unwelded $\alpha$-iron plates were evaluated, and then discussed the 20 - 30\% difference of crystallite size. The difference of $20 \%$ in analyzed values, which was caused by the $4.7 \mathrm{~K}$ change of $T_{\text {moderator }}$, possibly cause incorrect evaluation of the crystallite size. Therefore, the $4.7 \mathrm{~K}$ change of $T_{\text {moderator }}$ was found to be not acceptable for crystallite size analy- 
sis. In the analyzed values of texture, the difference was $2.5 \%$. The difference of texture was almost acceptable because larger than $20 \%$ difference was discussed in an example of texture analysis[22].

It was found that $T_{\text {moderator }}$ became almost stable after an hour operation in Fig. 4. The undesirable effect caused by $T_{\text {moderator }}$ change is expected to be avoided by excluding data before the moderator has warmed up. For confirmation of the effect of a gradual increase of $T_{\text {moderator }}$ (less than $1 \mathrm{~K}$ ) after the initial one hour, the other simulated $\operatorname{Tr}(E)$ which is calculated by the measured neutron spectra at $T_{\text {moderator }}$ of $302.0 \mathrm{~K}$ and that of $302.9 \mathrm{~K}$ was analyzed with RITS code. It was found that the difference of crystallite size between the simulated and theoretical $\operatorname{Tr}(E)$ was $3.8 \%$. This difference is almost acceptable because the difference of $3.8 \%$ is much smaller than the difference of $20-30 \%$, which was discussed in the crystallite size analysis[22]. The difference of texture in this case was $1.3 \%$, and this is acceptable because of the same reason above.

Although these results were obtained in the HUNS, the increase of $T_{\text {moderator }}$ is also concerned in other compact electron accelerator-driven thermal neutron sources, and the change of $T_{\text {moderator }}$ possibly cause incorrect evaluation in spectroscopic transmission analysis. Therefore, the monitoring of $T_{\text {moderator }}$ is recommended to avoid this effect and to guarantee the reliability of the spectroscopic transmission measurement especially in such neutron source.

\section{Conclusion}

We simultaneously measured neutron spectra and moderator temperature in the HUNS facility for 6 hours after the start of the accelerator operation. In this measurement, it was found that the neutron spectra shifted with the $4-5 \mathrm{~K}$ increase of the moderator temperature, and that the shape-change of the measured neutron spectrum was $\sim 3 \%$ at maximum. When there were $\sim 3 \%$ shape-change, it was found that crystal- 
lite size can be incorrectly evaluated in Bragg-edge analysis, for example. The effect of the moderator-temperature change possibly causes incorrect evaluation in spectroscopic transmission analysis. However, it was also found that the ratio of the neutron effective temperature and the moderator temperature was constant although both changed with the accelerator operation. This result indicated that the neutron effective temperature was well estimated by the moderator temperature. Additionally, it was also found that the changes of both temperatures were limited to be within the first hour. The adverse effect of the temperature change, being estimated to bring $\sim 3 \%$ discrepancy at maximum in the neutron transmission spectra in HUNS case, can be easily avoided by excluding data before the moderator warms up.

The increase of moderator temperature and undesirable effects from the shape-change of measured neutron spectra are also concerned in other compact electron acceleratordriven thermal neutron sources. Therefore, the monitoring of moderator temperature is recommended to guarantee the reliability of a measurement without sacrifices of cost, simplicity and maintainability in other compact electron accelerator-driven thermal neutron sources.

\section{Acknowledgement}

The authors thank Mr. Kohichi Sato of Hokkaido University for the LINAC operation and assistance with the experiment. This work was partially supported by JSPS KAKENHI Grant Number 17J01054.

\section{References}

[1] Gabriel TA, Haines JR, McManamy TJ, et al. Overview of the Spallation Neutron Source (SNS) with emphasis on target system. J. Nucl. Mater. 2003 May;318:1-13.

[2] Takada H, Haga K, Teshigawara M, et al. Materials and Life Science Experimental Facility at the 
Japan Proton Accelerator Research Complex I: Pulsed Spallation Neutron Source. Quantum Beam Sci. 2017 May;1:8.

[3] Lindroos M, Bousson S, Calaga R, et al. The European spallation source. Nucl. Instrum. Meth. B. 2011 Apr;269:3258-3260.

[4] Carpenter JM. ICANS to UCANS: Parallel evolution. Phys. Procedia. 2012;26:1-7.

[5] Kiyanagi Y. Neutron imaging at compact accelerator-driven neutron sources in Japan. J. Imaging. 2018 Mar;4:55.

[6] Furusaka M, Sato H, Kamiyama T, et al. Activity of Hokkaido University Neutron Source, HUNS. Phys. Procedia. 2014;60:167-174.

[7] Aomori Prefecture Quantum Science Center. [cited 2018 Oct 4]. Available from: https://www.aomoriqsc.jp/en/

[8] Otake Y. RIKEN compact neuron systems with fast and slow neutrons. Plasma Fusion Res. 2018 Oct;13:2401017.

[9] Tasaki S, Nagae T, Hirose M, et al. Properties and possible applications of Kyoto University Accelerator based Neutron Source (KUANS). Phys. Procedia. 2014;60:181-185.

[10] Takahashi Y, Kiyanagi Y, Watanabe K, et al. Development of a neutron source for imaging at the electron linac facility in Kyoto University Research Reactor Institue. Physica B. 2018 Jun. DOI:10.1016/j.physb.2018.06.009.

[11] SHI-ATEX Co.,Ltd. [cited 2018 Oct 4]. Available from: https://www.shi-atex.com

[12] Kiyanagi Y, Sato H, Kamiyama T, et al. A new imaging method using pulsed neutron sources for visualizing structual and dynamical information. J. Phys.: Conf. Ser. 2012;340:012010.

[13] Uno S, Uchida T, Sekimoto M, et al. Two-dimensional neutron detector with GEM and its applications. Phys. Procedia. 2012;26:142-152.

[14] Parker JD, Harada M, Hattori K, et al. Spatial resolution of a PIC-based neutron imaging detector. Nucl. Instrum. Meth. A. 2013 Jun; 726:155-161.

[15] Watanabe N. [Outline of spallation neutron source engineering]. JAERI-Review. 2000 Jan;00-031 [in 
Japanese].

[16] Sato H, Kamiyama T, Kiyanagi Y. A Rietveld-type analysis code for pulsed neutron Bragg-edge transmission imaging and quantitative evaluation of texture and microstructure of a welded $\alpha$-iron plate. Mater. Trans. 2011 May;52:1294-1302.

[17] Sato H, Kamiyama T, Iwase K, et al. Pulsed neutron spectroscopic imaging for crystallographic texture and microstructure. Nucl. Instrum. Meth. A. 2011 Sep;651:216-220.

[18] Sato H, Shinohara T, Kiyanagi R, et al. Upgrade of Bragg edge analysis techniques of the RITS code for crystalline structural information imaging. Phys. Procedia. 2013;43:186-195.

[19] Sato H, Sato T, Shiota Y, et al. Relation between Vickers hardness and Bragg-edge broadening in quenched steel rods observed by pulsed neutron transmission imaging. Mater. Trans. 2015 Jun;56:1147-1152.

[20] Beckurts KH, Wirtz K. Neutron Physics. Berlin Hidelberg: Springer-verlag; 1964. 10, Thermalization of neutrons; p.180-228.

[21] Japanese Industrial Standards (JIS). Thermocouples. Tokyo: JIS; 2015. Standard No. JIS C 1602:2015.

[22] Sato H. Deriving quantitative crystallographic information from the wavelength-resolved neutron transmission analysis performed in imaging mode. J. Imaging. 2017 Dec;4:7. 


\section{Figure Captions}

Figure 1 Experimental setup.

Figure 2 An example of the fitting analysis. In the upper graph, dots with error bars show a measured neutron energy spectrum, and the line shows a result of the fitting. The fitting range of the Maxwell-Boltzmann distribution (Eq. (1)) is between $0.01-0.07 \mathrm{eV}$, and that of Eq. (2) is between $0.07-10 \mathrm{eV}$. In the lower graph, the differences of the measured and fitting spectra are shown.

Figure 3 The neutron energy spectrum at $T_{\text {moderator }}$ of $298.2 \mathrm{~K}$, which was measured during the first 5 minutes from the measurement start, is shown by the dashed line, and that of $302.9 \mathrm{~K}$, which was measured during the last 5 minutes to measurement end, is shown by a solid line. The statistical errors of two spectra were smaller than $1 \%$ above the energy range of $0.01 \mathrm{eV}$.

Figure 4 The time evolution of the moderator temperature $\left(T_{\text {moderator }}\right)$ and effective neutron temperature $\left(T_{\text {neutron }}\right)$ with the accelerator operation time is shown in the upper graph, and that of the ratio of the two temperatures is shown in the lower graph. The errors of $T_{\text {neutron }}$ in the fitting analysis and the standard deviations of $T_{\text {moderator }}$ were shown as error bars.

Figure 5 The comparison of simulated and theoretical neutron transmission spec- 
tra of $5 \mathrm{~mm}$-thick $\alpha$-Fe. Dots and error bars show a simulation which represents the transmission spectrum with the change of $T_{\text {moderator }}$, and line shows the theoretical prediction. 


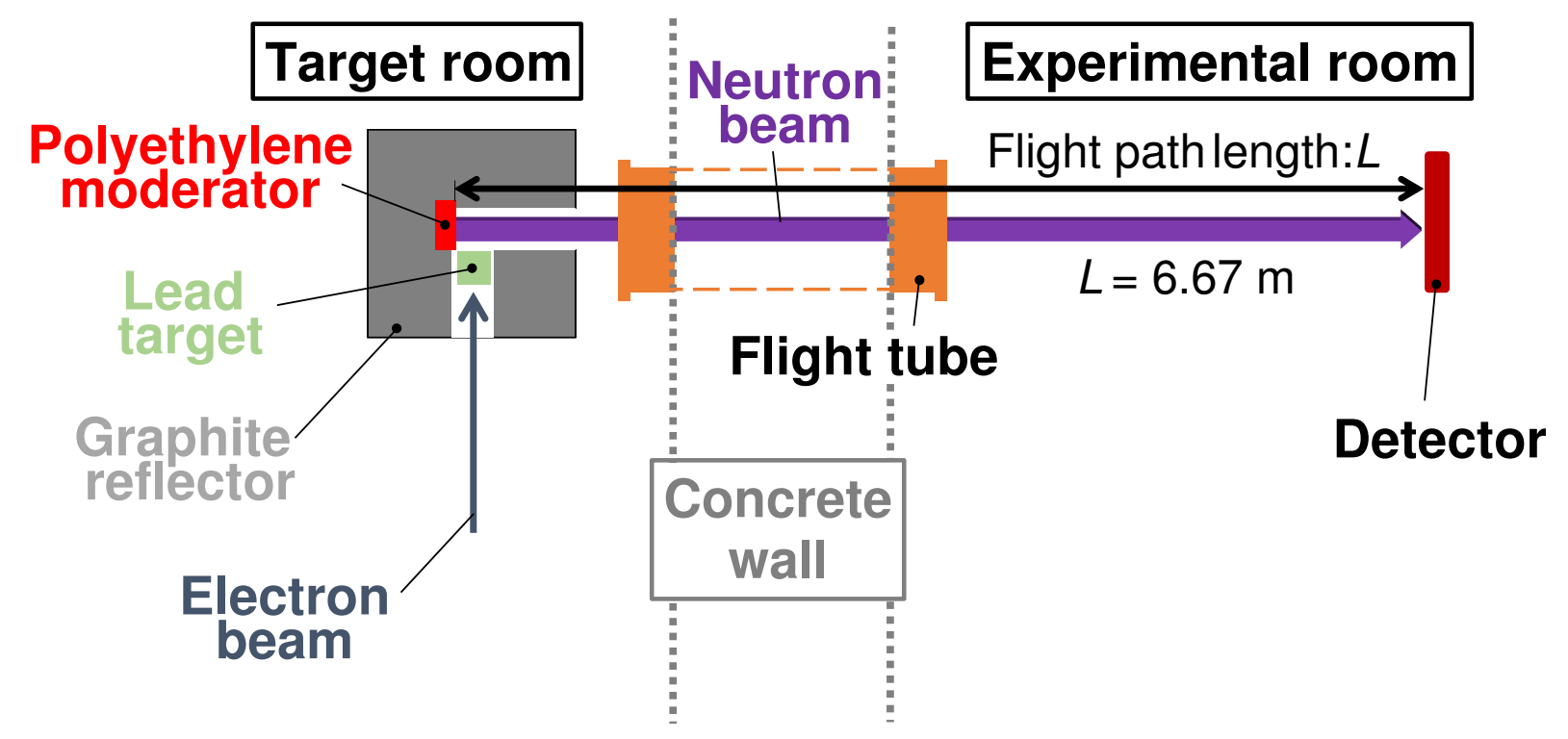

Figure 1 Experimental setup. 


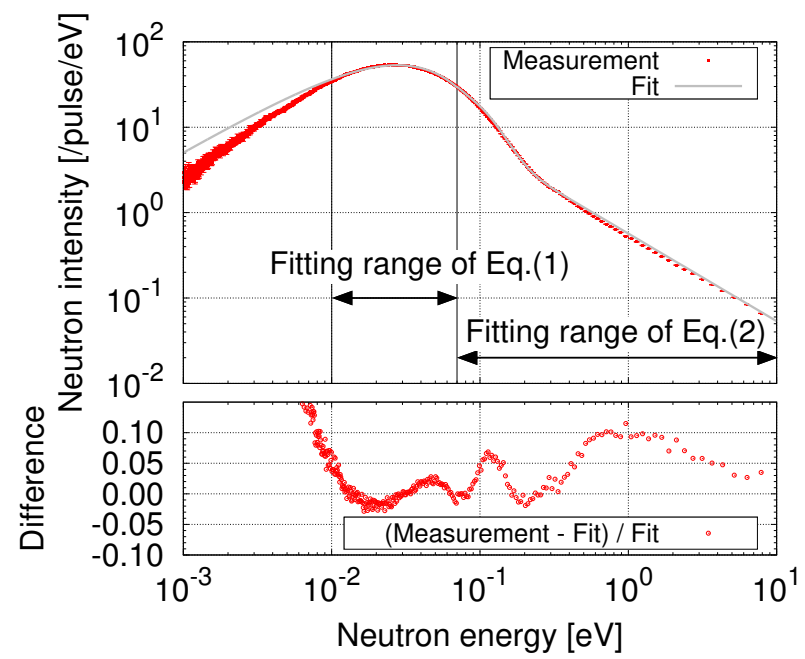

Figure 2 An example of the fitting analysis. In the upper graph, dots with error bars show a measured neutron energy spectrum, and the line shows a result of the fitting. The fitting range of the MaxwellBoltzmann distribution (Eq. (1)) is between $0.01-0.07 \mathrm{eV}$, and that of Eq. (2) is between $0.07-10$ $\mathrm{eV}$. In the lower graph, the differences of the measured and fitting spectra are shown. 


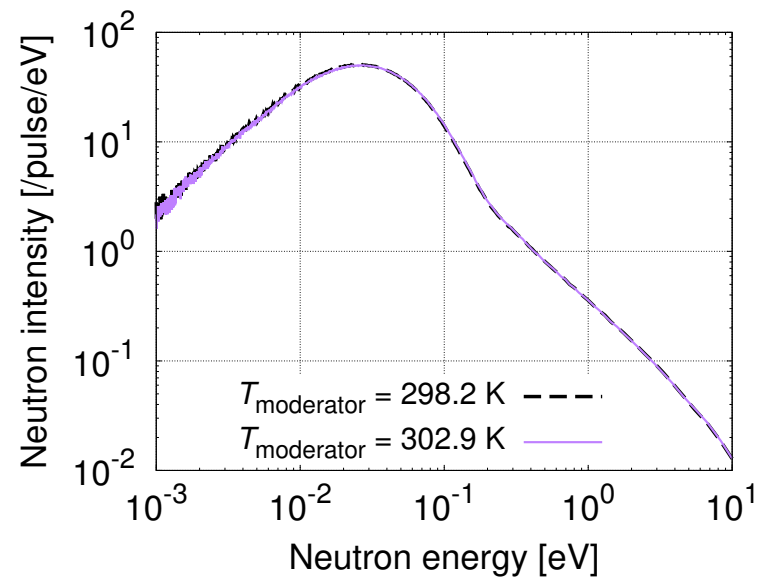

Figure 3 The neutron energy spectrum at $T_{\text {moderator }}$ of $298.2 \mathrm{~K}$, which was measured during the first 5 minutes from the measurement start, is shown by the dashed line, and that of $302.9 \mathrm{~K}$, which was measured during the last 5 minutes to measurement end, is shown by a solid line. The statistical errors of two spectra were smaller than $1 \%$ above the energy range of $0.01 \mathrm{eV}$. 


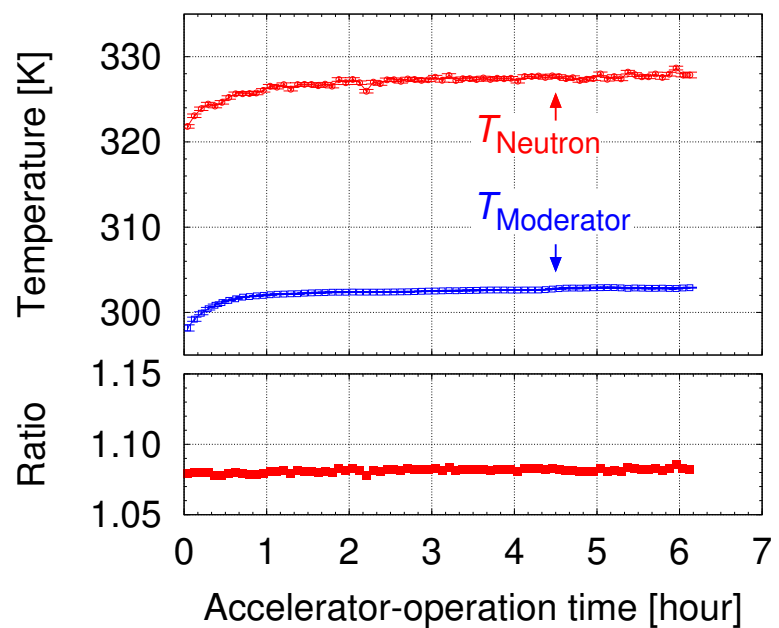

Figure 4 The time evolution of the moderator temperature $\left(T_{\text {moderator }}\right)$ and effective neutron temperature $\left(T_{\text {neutron }}\right)$ with the accelerator operation time is shown in the upper graph, and that of the ratio of the two temperatures is shown in the lower graph. The errors of $T_{\text {neutron }}$ in the fitting analysis and the standard deviations of $T_{\text {moderator }}$ were shown as error bars. 


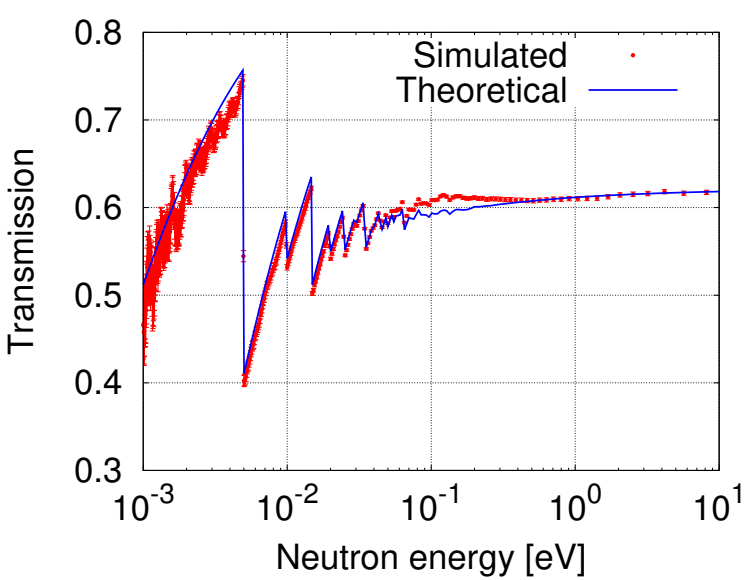

Figure 5 The comparison of simulated and theoretical neutron transmission spectra of 5 mm-thick $\alpha$-Fe.

Dots and error bars show a simulation which represents the transmission spectrum with the change of $T_{\text {moderator }}$, and line shows the theoretical prediction. 